\title{
Archivos de Cardiología de México

\section{Factores de riesgo asociados a la cirugía del adulto con cardiopatía congénita: 22 años de experiencia. ¿Quién debe intervenirlos?}

\author{
Ángel Aroca ${ }^{a, *}$, Luz Polo ${ }^{a}$, Napoleón Pérez-Farinós ${ }^{b}$, Ana E. González ${ }^{c}$, \\ Montserrat Bret ${ }^{d}$, Elizabeth Aguilar ${ }^{\mathrm{e}}$ y José M. Oliver ${ }^{\mathrm{c}}$
}

\author{
a Servicio de Cirugía Cardíaca Infantil y Unidad de Cardiopatías Congénitas del Adulto, Hospital Universitario La Paz, Madrid, \\ España \\ b Departamento de Medicina Preventiva y Salud Pública (Historia de la Ciencia), Universidad Complutense de Madrid, Madrid, \\ España \\ c Servicio de Cardiología y Unidad de Cardiopatías Congénitas del Adulto, Hospital Universitario La Paz, Madrid, España \\ ' Servicio de Radiodiagnóstico, Hospital Universitario La Paz, Madrid, España \\ e Servicio de Cirugía Cardiovascular, Hospital Centenario Miguel Hidalgo, Aguascalientes, México
}

Recibido el 15 de agosto de 2013; aceptado el 5 de febrero de 2014

\author{
PALABRAS CLAVE \\ Cardiopatías \\ congénitas; \\ Adultos; \\ Cirugía cardíaca; \\ Factores de riesgo; \\ Mortalidad; \\ España
}

\begin{abstract}
Resumen
Objetivo: Analizar la asociación entre mortalidad en cirugía de cardiopatías congénitas del adulto y los factores relacionados con el paciente y la intervención.

Método: Estudio descriptivo de intervenciones por cirujanos con actividad habitual en cardiopatías congénitas (238), cardiopatías adquiridas (117) y residentes (108). Se evaluó la asociación de la mortalidad con el riesgo y complejidad quirúrgica, actividad habitual del cirujano, y tiempo de circulación extracorpórea y de pinzamiento aórtico, mediante modelos de regresión logística.

Resultados: Se incluyeron 463 cirugías (442 con circulación extracorpórea) entre 1991 y 2012. Edad mediana de intervención: 34 años (52.8\% mujeres); primera cirugía 295 , reintervención 168. La puntuación mediana del Aristóteles fue 6.8, con complejidad significativamente mayor tras reestructurarse la Unidad en 2001. La mortalidad hospitalaria total fue del 3.9\%. La mortalidad se asoció significativamente al número de intervenciones previas (OR: 5.02; IC 95\%: 1.44-17.52), intervenciones por cirujanos de cardiopatía adquirida (OR: 3.53; IC 95\%: 1.1410.98), Aristóteles alto (OR: 1,64; IC 95\%: 1.18-2.29), y tiempos prolongados de extracorpórea (OR: 1.13; IC 95\%: 1.07-1.19).

Conclusiones: La mortalidad en cirugía de cardiopatía congénita en adultos es baja. Las intervenciones de alta complejidad, tiempos elevados de extracorpórea y múltiples reintervenciones
\end{abstract}

\footnotetext{
* Autor para correspondencia: Paseo de la Castellana 261, 28046 Madrid. Teléfono: +34629277743.

Correo electrónico: aarocap@telefonica.net (Á. Aroca).
} 


\section{KEYWORDS}

Congenital heart

defects;

Adults;

Cardiac surgery;

Mortality;

Risk factors;

España se asocian con mayor mortalidad. La participación de cirujanos especialistas en cardiopatías congénitas se asocia con mejores resultados.

(c) 2013 Instituto Nacional de Cardiología Ignacio Chávez. Publicado por Masson Doyma México S.A. Todos los derechos reservados.

Risk factors for surgery of congenital heart disease in adults. Twenty-two years of experience. Who should operate them?

\begin{abstract}
Objective: To assess the association between mortality in surgery of congenital heart disease in adults, and factors related to patients and operations.

Method: Descriptive study of operations performed by specialized surgeons in congenital heart surgery (238), adult acquired surgery (117), and specialty residents (108). The association of mortality with surgical risk and complexity, specialization of surgeon, cardiopulmonary by-pass and aortic cross clamping was assessed fitting logistic regression models.

Results: A total of 463 operations were included (442 with cardiopulmonary by-pass) in the study performed between 1991 and 2012. Median age at surgery: 34; 52.8\% were women. First surgery: 295, reoperation: 168 . Median score of Aristotle was 6.8 , with significantly higher complexity since 2001, after restructuring the Unit. Overall hospital mortality was $3.9 \%$. Mortality was significantly associated to number of previous surgeries (OR: $5.02 ; 95 \% \mathrm{Cl}: 1.44-17.52$ ), operations by acquired heart disease surgeons (OR: 3.53; $95 \% \mathrm{Cl}: 1.14-10.98)$, higher Aristotle (OR: 1,64; $95 \% \mathrm{Cl}: 1.18-2.29)$, and high cardiopulmonary by-pass time (OR: $1.13 ; 95 \% \mathrm{Cl}: 1.07-1.19)$. Conclusions: Surgery of congenital heart disease in adults has been performed with low mortality. High complexity interventions, prolonged cardiopulmonary by-pass times and multiple reoperations were associated to higher mortality. Participation of cardiac surgeons specialized in congenital heart disease is associated with better outcomes.

(c) 2013 Instituto Nacional de Cardiología Ignacio Chávez. Published by Masson Doyma México S.A. All rights reserved.
\end{abstract}

\section{Introducción}

En países desarrollados, con baja natalidad, los pacientes adultos con cardiopatía congénita («congénitos adultos») superan a la población infantil con dichas enfermedades ${ }^{1,2}$. Entre los motivos por los que estos pacientes pueden ser intervenidos en la edad adulta se encuentran: secuelas y/o lesiones residuales de una cardiopatía operada en la infancia, cardiopatías solo paliadas cuya reparación se completa en la edad adulta, otras que por su leve repercusión funcional se diagnostican tardíamente, o por aparición de una cardiopatía adquirida en portadores de una cardiopatía congénita ${ }^{3,4}$. En la última década distintos grupos han aportado datos sobre los resultados de dichas intervenciones y han buscado factores relacionados con la mortalidad $^{5-8}$.

Si bien los congénitos adultos suponen un porcentaje creciente en la actividad de los servicios de cirugía cardíaca ${ }^{9,10}$, en España, la información sobre la cuantía, complejidad y los resultados de dichas intervenciones es escasa. Recientemente se ha intentado determinar cuál es el ámbito ideal en el que deben ser operados estos pacientes y si los resultados mejoran cuando son tratados por cirujanos dedicados habitualmente a esta actividad ${ }^{11-15}$.

El presente es un estudio descriptivo de las operaciones realizadas a sujetos mayores de 18 años con cardiopatías congénitas, relacionando la indicación quirúrgica con la técnica quirúrgica, clasificándolos en categorías como propone el Registro Europeo de Cirugía Cardiotorácica Congénita, para determinar si existe asociación entre la mortalidad hospitalaria y ciertos factores inherentes al paciente y a la cirugía. Con este análisis se pretende dar una respuesta al debate actual de quién debe realizar las intervenciones de estos enfermos.

\section{Métodos}

Se trata de un estudio descriptivo de 463 intervenciones mayores, llevadas a cabo sobre 432 pacientes (un mismo enfermo puede operarse varias veces) en la Unidad de Cardiopatías Congénitas del Adulto (UCCA) de nuestro hospital, entre los años 1991 y 2012.

En las operaciones participaron 16 cirujanos de plantilla, 7 con actividad habitual en cardiopatías adquiridas, que realizaron 117 operaciones (mínimo: una; máximo: 79) y 9 con actividad habitual en cardiopatías congénitas, con 238 intervenciones (mínimo: una; máximo: 158). Las 108 intervenciones restantes, de menor complejidad, fueron realizadas por médicos internos residentes, supervisadas por al menos un cirujano de plantilla. 
Para cada operación se recogió información sobre edad, sexo, número de intervenciones previas, actividad habitual del cirujano que intervino, clasificación según escala de riesgo quirúrgico RACHS-1 (6 categorías de riesgo, desde mortalidad del $0.4 \%$ para la categoría 1 a la que pertenece, por ejemplo, la reparación de una comunicación interauricular, hasta el $47.7 \%$ de la categoría 6$)^{16}$ y de complejidad Aristóteles básico (puntuación para las cirugías cardiotorácicas mayores entre 3 , la más sencilla, como la reparación de una comunicación interauricular tipo ostium secundum y 15 la más compleja $)^{17}$, tiempo de circulación extracorpórea (CEC) y de pinzamiento aórtico (ClAo). Se realizó la prueba de la $t$ de Student para evaluar si los tiempos de CEC y de $\mathrm{ClAo}$, y la estancia postoperatoria hospitalaria eran significativamente distintos entre los pacientes operados por vez primera y los reoperados. También se evaluó si la estancia postoperatoria hospitalaria en cada intervención tenía asociación con la complejidad (Aristóteles básico), mediante el coeficiente de correlación de Spearman.

Además de la estancia postoperatoria hospitalaria, se consideraron marcadores de calidad de los procedimientos quirúrgicos, y se calcularon los tiempos de intubación orotraqueal y de estancia en reanimación postoperatoria. Se realizó un análisis univariante para evaluar la asociación de la mortalidad con los tiempos de CEC y ClAo, el número de intervenciones previas, la puntuación en las escalas de riesgo quirúrgico (RACHS-1) y complejidad (Aristóteles básico) y la actividad habitual del cirujano (cardiopatía adquirida o congénita). Asimismo, se evaluó si había diferencias significativas en las escalas de riesgo quirúrgico (RACHS-1) y complejidad (Aristóteles básico), y si la operación era o no reintervención, entre los cirujanos de actividad habitual en cardiopatías congénitas y el resto de los cirujanos (excluyendo a los residentes). Se utilizó la prueba de la Chi-cuadrado en variables cualitativas y la t de Student en caso de cuantitativa continua.

Para estudiar la asociación de la mortalidad con las variables de estudio se ajustaron 2 modelos de regresión logística. En el primer modelo la variable dependiente fue la mortalidad entre los pacientes, y las variables independientes se seleccionaron cuando mostraron asociación significativa $(p<0.05)$ en el análisis univariante. En este modelo se incluyeron las intervenciones realizadas por los residentes, puesto que solo se evaluaron las características de los pacientes y las intervenciones.

En el segundo modelo, en el que la variable dependiente también fue la mortalidad entre los pacientes, se excluyeron los pacientes que habían sido intervenidos por cirujanos residentes, todos sin mortalidad, para determinar la asociación de esta última con el ámbito de actuación habitual del cirujano de plantilla (cardiopatías congénitas o no). Además, se incluyeron aquellas variables que mostraron asociación estadísticamente significativa $(\mathrm{p}<0.05)$ en el análisis univariante. Ambos modelos fueron ajustados por sexo.

Para evaluar la progresión en la casuística y la experiencia creciente de los cirujanos se analizó si la complejidad quirúrgica media medida a través del Aristóteles básico variaba significativamente con el tiempo, mediante el coeficiente de correlación de Spearman.

En el año 2001 se produjeron cambios organizativos en la UCCA orientados hacia un protagonismo casi completo de los cirujanos con actividad habitual en cardiopatías congénitas.
Para determinar si dicha reestructuración tuvo impacto en los resultados quirúrgicos se evaluaron 2 indicadores antes y después de ese año. Se evaluó mediante la prueba de la t de Student si la complejidad de las intervenciones (medida a través de la mediana del Aristóteles) era significativamente distinta antes o después del año 2001. Y se evaluó si el porcentaje de reintervenciones era significativamente distinto a partir del año 2001, mediante la prueba de la Chi-cuadrada.

\section{Resultados}

Se efectuaron 463 intervenciones en 432 pacientes, de los que el $52.8 \%$ eran mujeres. La edad mediana en el momento de la intervención era de 34 años (mínimo: 18; máximo: 77). Se utilizó CEC en 442 intervenciones (95.5\%) y 21 se realizaron sin CEC.

Fueron primera cirugía 295 operaciones (63.7\%), en su mayoría correspondieron a formas leves de cardiopatías congénitas, desapercibidas hasta la vida adulta. Las 168 restantes fueron reintervenciones (36.3\%). De los reoperados, 101 tenían una sola intervención previa, 48 tenían 2 cirugías previas, 11 tenían 3, 6 tenían 4 operaciones y 2 tenían 5 intervenciones previas.

El tiempo medio de CEC fue de 107 min (DE: 70). Entre los pacientes operados por vez primera el tiempo medio de CEC fue de $82 \mathrm{~min}$ (DE: 46), y entre los reoperados, de $149 \mathrm{~min}$ (DE: 82); la diferencia entre ambos tiempos fue estadísticamente significativa $(p<0.001)$. El tiempo medio de ClAo fue de $61 \mathrm{~min}$ (DE: 44). Entre los pacientes operados por vez primera el tiempo medio de ClAo fue de $55 \mathrm{~min}$ (DE: 34), mientras que entre los reoperados fue de $71 \mathrm{~min}$ (DE: 56); la diferencia entre ambos tiempos también resultó estadísticamente significativa $(p<0.001)$.

La estancia media postoperatoria hospitalaria fue de 12.6 días (DE: 9.6), y se encontró una correlación estadísticamente significativa entre ella y la complejidad quirúrgica (Aristóteles) $(r=0.27, p<0.001)$. La estancia media postoperatoria hospitalaria entre los pacientes operados por primera vez fue de 10.8 días (DE: 6.8), mientras que en los reoperados fue de 14.8 días (DE: 11.9 ); la diferencia entre ambos tiempos de estancia fue estadísticamente significativa $(p=0.002)$.

El tiempo mediano de estancia en la UVI fue de 3 días (mínimo: uno; máximo: 68). El tiempo mediano de intubación orotraqueal fue de $7 \mathrm{~h}$ (mínimo: una; máximo: 1632). No se hallaron diferencias estadísticamente significativas en ninguno de dichos tiempos entre las reoperaciones y las que eran primera intervención.

El RACHS-1 y el Aristóteles básico mostraron una alta correlación (coeficiente de correlación $\rho$ de Spearman: 0.77; $p<0.001$ ), por lo que no se utilizaron las 2 escalas simultáneamente para ajustar el modelo multivariante. Según RACHS-1, el $35.7 \%$ de los pacientes pertenecían a la categoría de riesgo 1 , el $22.6 \%$ a la 2 , el $41.3 \%$ a la 3 y el $0.4 \%$ a la categoría de riesgo 4. En la escala Aristóteles básico, la puntuación mediana fue de 6.8 (mínimo: 3; máximo: 12.5).

Los diagnósticos con los que los pacientes fueron llevados a quirófano, la indicación de intervención y si el paciente falleció se recogen de modo esquemático en la tabla 1, y de forma completa en la tabla 2. 
Tabla 1 Agrupación de los pacientes de la serie por indicación quirúrgica y fallecidos en cada grupo

\begin{tabular}{lcc}
\hline Diagnóstico-indicación & $\mathrm{n}$ & Mortalidad (n) \\
\hline Defectos septales primarios/residuales & 152 & 1 \\
Anomalías de venas pulmonares/sistémicas & 31 & 0 \\
Arterias y venas torácicas & 32 & 2 \\
Ventrículo único & 16 & 2 \\
Transposición de grandes vasos & 6 & 1 \\
Lesiones de corazón izquierdo aisladas & 54 & 3 \\
Lesiones de corazón izquierdo + defecto septal & 20 & 0 \\
Lesiones de corazón izquierdo + arterias y venas torácicas & 14 & 2 \\
Lesiones de corazón izquierdo a 2 niveles & 7 & 2 \\
Lesiones de corazón izquierdo y derecho & 17 & 2 \\
Lesiones de corazón derecho aisladas & 40 & 1 \\
Lesiones de corazón derecho + defecto septal & 59 & 1 \\
Lesiones de corazón derecho a 2 niveles & 15 & 1 \\
\hline
\end{tabular}

La mortalidad hospitalaria referida al total de procedimientos fue del $3.9 \%$. Fallecieron 18 pacientes. El porcentaje de mortalidad según las variables estudiadas puede verse en la tabla 3. De los 295 casos que eran primera cirugía fallecieron 7 (2.4\%). En las 168 cirugías que tenían una o más intervenciones previas se produjeron 11 fallecimientos (7\%).

La mortalidad en las operaciones con CEC fue del 4.1\%. No hubo mortalidad en las 21 cirugías sin CEC. El tiempo medio de CEC fue $94 \mathrm{~min}$ mayor en los pacientes que fallecieron $(p=0.09)$. Asimismo, el tiempo medio de ClAo fue $43 \mathrm{~min}$ mayor en los individuos fallecidos $(p=0.029)$.

La mortalidad en las intervenciones llevadas a cabo por cirujanos cuya actividad quirúrgica habitual es la de las malformaciones congénitas fue del $4.2 \%$, mientras que la mortalidad en las intervenciones por cirujanos que no se dedican específicamente a congénitas fue del $6.8 \%$, siendo esta diferencia estadísticamente significativa $(p=0.041)$.

En la tabla 4 puede verse que los cirujanos con actividad habitual en cardiopatías congénitas realizaban intervenciones más complejas, según la escala Aristóteles, y también más reoperaciones, que los cirujanos con actividad habitual en cardiopatías adquiridas.

Los resultados obtenidos en los análisis multivariante pueden verse en las tablas 5 y 6 . Los pacientes que fueron intervenidos 3 o más veces tenían una mortalidad significativamente mayor que los no intervenidos previamente 0 un máximo de 2 veces (OR: 5.02; IC 95\%: 1.44-17.52). Valores elevados de Aristóteles se asociaron a mayor mortalidad (OR: 1.64; IC 95\%: 1.18-2.29). La mortalidad fue 3.53 veces mayor si los cirujanos que intervenían no tenían su ámbito de actuación habitual en las cardiopatías congénitas (IC 95\%: 1.14-10.98). Además, cada $10 \mathrm{~min}$ de aumento en el tiempo de CEC se incrementaba significativamente la mortalidad (OR: 1.13; IC 95\%: 1.07-1.19).

Cuanto más reciente (porción más moderna de la serie) era el año de la intervención, mayor fue la complejidad quirúrgica medida con el Aristóteles básico (coeficiente de correlación de Spearman: 0.17; $\mathrm{p}<0.001$ ). Además, la complejidad quirúrgica media a partir del año 2001 fue 0.97 puntos mayor que antes de dicho año $2001(p<0.001)$. El porcentaje de reintervenciones hasta el año 2001 fue del
23.9\%, mientras que a partir de 2001 fue del $44.4 \%\left(\chi^{2}\right.$ : 19.80; $\mathrm{p}<0.001$ ) (fig. 1).

Se produjeron complicaciones postoperatorias (morbilidad) en 70 de los 463 procedimientos mayores realizados (15\%). El más frecuente fue la aparición transitoria o definitiva de una arritmia supraventricular. La descripción completa de la morbilidad perioperatoria se recoge en la tabla 7.

\section{Discusión}

La cirugía de las cardiopatías congénitas del adulto constituyen un porcentaje importante de la actividad de un servicio de cirugía cardíaca donde existe una UCCA consolidada como es el caso de nuestro hospital, donde se realizan unas 500 cirugías cardiotorácicas mayores anuales ( 300 sobre cardiopatía adquirida y 200 sobre congénita), y donde como media trabajan 10 cirujanos de plantilla. Este volumen de

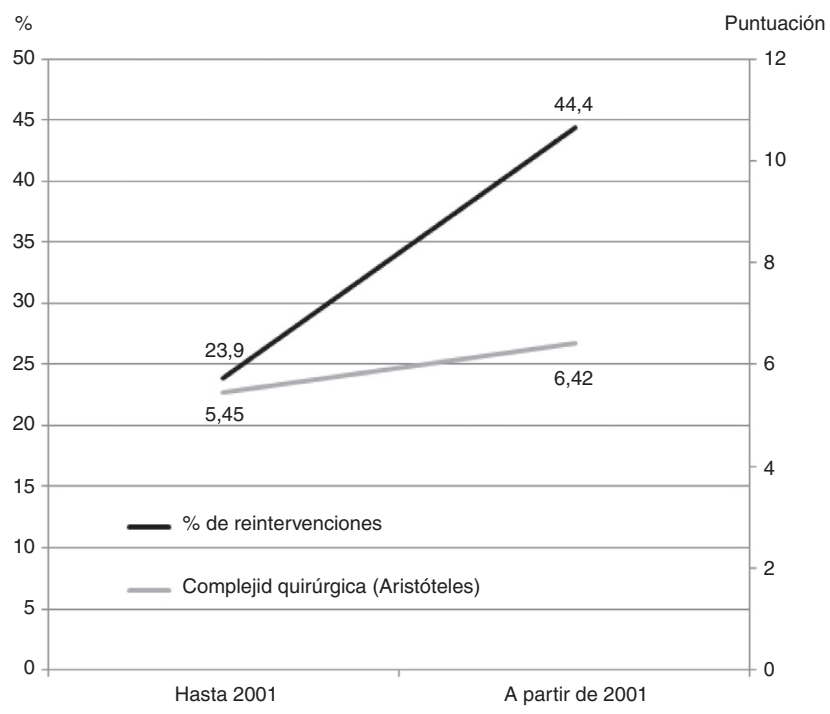

Figura 1 Impacto de la reestructuración del Servicio de Cirugía Cardíaca del Hospital La Paz en 2001. Porcentaje de reintervenciones y complejidad quirúrgica (Aristóteles). 
Tabla 2 Serie completa de los procedimientos realizados, clasificados por indicación quirúrgica, y fallecidos en cada grupo

\begin{tabular}{|c|c|c|c|c|c|c|}
\hline Diagnóstico-indicación & $\mathrm{n}$ & Técnica quirúrgica principal & $\mathrm{n}$ & Procedimiento asociado & $\mathrm{n}$ & Mortalidad (n) \\
\hline Defectos septales & 152 & & & & & 1 \\
\hline \multirow[t]{3}{*}{ CIA primaria/residual/FOP } & 126 & Cierre CIA & 126 & Retirada Amplatzer & 5 & 0 \\
\hline & & & & Cirugía arritmia/MP & 6 & 0 \\
\hline & & & & Puente coronario & 3 & 0 \\
\hline \multirow[t]{2}{*}{ Heterotaxias septadas } & 2 & Septación con parche & 2 & Plastia mitral & 2 & 0 \\
\hline & & & & Plastia tricuspídea & 2 & 0 \\
\hline \multirow[t]{3}{*}{ CIV primaria/residual } & 12 & Cierre CIV & 12 & Cierre DAP & 1 & 0 \\
\hline & & & & Debanding & 1 & 0 \\
\hline & & & & Cierre FOP & 2 & 0 \\
\hline DPSAV & 12 & Reparación completa & 12 & & & 1 \\
\hline $\begin{array}{l}\text { Anomalias de venas } \\
\text { pulmonares/sistémicas }\end{array}$ & 31 & & & & & 0 \\
\hline Cor triatriatum & 2 & Reparación completa & 2 & Cirugía arritmias & 1 & 0 \\
\hline \multirow[t]{2}{*}{$\mathrm{DVPAP}+\mathrm{CIA}$} & 27 & $\begin{array}{l}\text { Reparación con túnel } \\
\text { intracava o Warden }\end{array}$ & 27 & Plastia tricuspídea & 3 & 0 \\
\hline & & & & Recambio MP & 1 & 0 \\
\hline $\begin{array}{l}\text { Miscelánea retorno venoso } \\
\text { sistémico/pulmonar }\end{array}$ & 2 & Conducto & 2 & & & 0 \\
\hline Arterias y venas torácicas & 32 & & & & & 2 \\
\hline DAP & 3 & Oclusión DAP & 3 & & & 0 \\
\hline \multirow[t]{3}{*}{ CoA sin CEC } & 13 & Sección y sutura & 2 & $\begin{array}{l}\text { Implante electrodo MP } \\
\text { en VI para } \\
\text { resincronizador }\end{array}$ & 1 & 0 \\
\hline & & Conducto & 5 & & & 0 \\
\hline & & Aortoplastia con parche & 6 & & & 0 \\
\hline $\begin{array}{l}\text { ReCoA + hipoplasia arco } \\
\text { con CEC }\end{array}$ & 2 & Conducto AoA-AoD & 2 & & & 0 \\
\hline $\begin{array}{l}\text { Origen anómalo coronaria } \\
\text { izquierda desde aorta }\end{array}$ & 2 & By pass AMI & 2 & & & 0 \\
\hline $\begin{array}{l}\text { Aneurisma Ao (primario } \\
\text { o secuela) }\end{array}$ & 7 & Conducto & 7 & & & 0 \\
\hline Seudoaneurisma Ao & 5 & Conducto & 7 & & & 2 \\
\hline Ventrículo único & 16 & & & & & 2 \\
\hline Heterotaxia + DVPAT & 1 & Conexión DVPAT a Al + Glenn & 1 & & & 1 \\
\hline \multirow{2}{*}{$\begin{array}{l}\text { Situación ventrículo único } \\
\text { compensado o paliado } \\
\text { primer estadio }\end{array}$} & 8 & Glenn bidireccional & 7 & $\begin{array}{l}\text { SVA por endocarditis } \\
\text { aguda }\end{array}$ & 1 & 0 \\
\hline & & Glenn unidireccional & 1 & & & 0 \\
\hline $\begin{array}{l}\text { Situación ventrículo único } \\
\text { compensado o paliado } \\
\text { segundo estadio }\end{array}$ & 2 & Fontan extracardíaco & 2 & & & 0 \\
\hline Fontan fracasado & 4 & Conversión Fontan & 4 & & & 0 \\
\hline Fontan convertido fracasado & 1 & $\begin{array}{l}\text { Fenestración Fontan } \\
\text { convertido }\end{array}$ & 1 & ECMO & 1 & 1 \\
\hline Transposición grandes Vasos & 6 & & & & & 1 \\
\hline TGA septo intacto & 1 & Reparación tipo Senning & 1 & & & 0 \\
\hline $\begin{array}{l}\text { TGA septo intacto-Mustard, } \\
\text { dehiscencia túnel cavas }\end{array}$ & 1 & $\begin{array}{l}\text { Reparación CIA residual tipo } \\
\text { «seno coronario sin techo» }\end{array}$ & 1 & & & 0 \\
\hline L-TGA + AP + doble BT & 1 & Refístula BT & 1 & & & 1 \\
\hline $\begin{array}{l}\text { L-TGA + insuficiencia válvula } \\
\text { AV sistémica }\end{array}$ & 2 & SVM & 2 & & & 0 \\
\hline L-TGA + Rastelli & 1 & $\begin{array}{l}\text { Recambio conducto } \\
\text { ventrículo subpulmonar-AP }\end{array}$ & 1 & Cierre CIV residual & 1 & 0 \\
\hline $\begin{array}{l}\text { Lesiones de corazón izquierdo } \\
\text { aisladas }\end{array}$ & 50 & & & & & 3 \\
\hline
\end{tabular}


Tabla 2 (continuación)

\begin{tabular}{|c|c|c|c|c|c|c|}
\hline Diagnóstico-indicación & $\mathrm{n}$ & Técnica quirúrgica principal & $\mathrm{n}$ & Procedimiento asociado & $\mathrm{n}$ & Mortalidad (n) \\
\hline \multirow{2}{*}{$\begin{array}{l}\text { IM congénita/posreparación } \\
\text { DSAV }\end{array}$} & 8 & SVM & 7 & & & 1 \\
\hline & & Plastia mitral & 1 & & & 0 \\
\hline EM congénita & 2 & SVM & 2 & & & 0 \\
\hline DLA congénita & 6 & SVA & 6 & & & 0 \\
\hline \multicolumn{7}{|l|}{ posvalvulotomía } \\
\hline $\begin{array}{l}\text { EA congénita primaria } \\
\text { o posvalvulotomía }\end{array}$ & 7 & SVA & 7 & $\begin{array}{l}\text { Ampliación anillo } \\
\text { posterior }\end{array}$ & 1 & 0 \\
\hline \multirow{2}{*}{$\begin{array}{l}\text { IA congénita primaria } \\
\text { o residual }\end{array}$} & 6 & SVA & 4 & & & 0 \\
\hline & & Plastia aórtica & 2 & & & 0 \\
\hline EA subvalvular & 7 & $\begin{array}{l}\text { Resección EA } \\
\text { subvalvular } \pm \text { miectomía }\end{array}$ & 7 & & & 1 \\
\hline Aneurisma seno Valsalva & 1 & Reparación ASV & 1 & & & 0 \\
\hline $\begin{array}{l}\text { EA valvular y subvalvular } \\
\text { congénitas }\end{array}$ & 7 & SVA & 7 & $\begin{array}{l}\text { Resección } \\
M b+\text { miectomía septal }\end{array}$ & 7 & 1 \\
\hline \multirow{2}{*}{$\begin{array}{l}\text { EA residual congénita } \\
\text { multinivel }\end{array}$} & 2 & Bentall-Konno & 1 & & & \\
\hline & & SVA & 1 & $\begin{array}{l}\text { Ampliación anillo } \\
\text { posterior }\end{array}$ & 1 & 0 \\
\hline \multirow[t]{2}{*}{ IA o DLA + EA subvalvular } & 8 & SVA & 5 & $\begin{array}{l}\text { Ampliación } \\
\text { anillo + miectomía }\end{array}$ & 5 & 0 \\
\hline & & Plastia aórtica & 3 & $\begin{array}{l}\text { Resección } \\
\text { Mb + miectomía }\end{array}$ & 3 & 0 \\
\hline $\begin{array}{l}\text { Lesiones corazón } \\
\quad \text { izquierdo + defecto septal }\end{array}$ & 20 & & & & & 0 \\
\hline $\mathrm{EA}+\mathrm{CIA}$ & 1 & SVA & 1 & Cierre CIA & 1 & 0 \\
\hline $\mathrm{IA}+\mathrm{CIA}$ & 1 & Plastia aórtica & 1 & Cierre $\mathrm{CIA}$ & 1 & 0 \\
\hline $\mathrm{EM}+\mathrm{CIA}$ & 4 & Comisurotomía mitral & 4 & Cierre CIA & 4 & 0 \\
\hline $\mathrm{IM}+\mathrm{CIA}$ & 3 & Plastia mitral & 3 & Cierre $\mathrm{CIA}$ & 3 & 0 \\
\hline EA subvalvular + CIV & 3 & Resección Mb & 3 & Cierre CIV & 3 & 0 \\
\hline \multirow[t]{2}{*}{$\mathrm{IA}+\mathrm{CIV}$} & 5 & SVA & 3 & Cierre CIV & 5 & 0 \\
\hline & & Plastia aórtica & 2 & & & \\
\hline $\mathrm{DLA}+\mathrm{CIV}$ & 2 & SVA & 2 & Cierre CIV & 2 & 0 \\
\hline $\begin{array}{l}\text { Aneurisma seno } \\
\text { Valsalva + CIV + anillo } \\
\text { vascular }\end{array}$ & 1 & Cierre ASV + plastia aórtica & 1 & $\begin{array}{l}\text { Cierre CIV + sección } \\
\text { anillo vascular }\end{array}$ & 1 & 0 \\
\hline $\begin{array}{l}\text { Lesiones de corazón } \\
\text { izquierdo + arterias y venas } \\
\text { torácicas }\end{array}$ & 14 & & & & & 2 \\
\hline \multirow{3}{*}{$\begin{array}{l}\text { IA + aneurisma AoA post-CoA } \\
\text { reparada/Ross/conotruncal } \\
\text { reparada }\end{array}$} & 9 & SVA + recambio AoA & 2 & & & 0 \\
\hline & & Bentall & 5 & Cierre CIV & 1 & 1 \\
\hline & & David & 2 & & & 0 \\
\hline \multirow{2}{*}{$\begin{array}{l}\text { DLA + aneurisma de AoA } \\
\text { posvalvulotomía aórtica }\end{array}$} & 4 & SVA + recambio AoA & 3 & & & 1 \\
\hline & & Bentall & 1 & & & 0 \\
\hline $\begin{array}{l}\text { EA + aneurisma de AoA } \\
\text { posvalvulotomía aórtica }\end{array}$ & 1 & SVA + recambio AoA & 1 & & & 0 \\
\hline $\begin{array}{l}\text { Lesiones corazón izquierdo } \\
\text { a } 2 \text { niveles }\end{array}$ & 7 & & & & & 2 \\
\hline Shone con EM + EA & 1 & SVM + conducto apicoaórtico & 1 & & & 1 \\
\hline Aneurisma seno Valsalva + IA & 1 & SVA & 1 & Cierre ASV & & 0 \\
\hline \multirow[t]{2}{*}{ IM + EA subvalvular } & 4 & Plastia mitral & 3 & $\begin{array}{l}\text { Resección } \\
M b+\text { miectomía }\end{array}$ & 4 & 0 \\
\hline & & SVM & 1 & & & 0 \\
\hline
\end{tabular}


Tabla 2 (continuación)

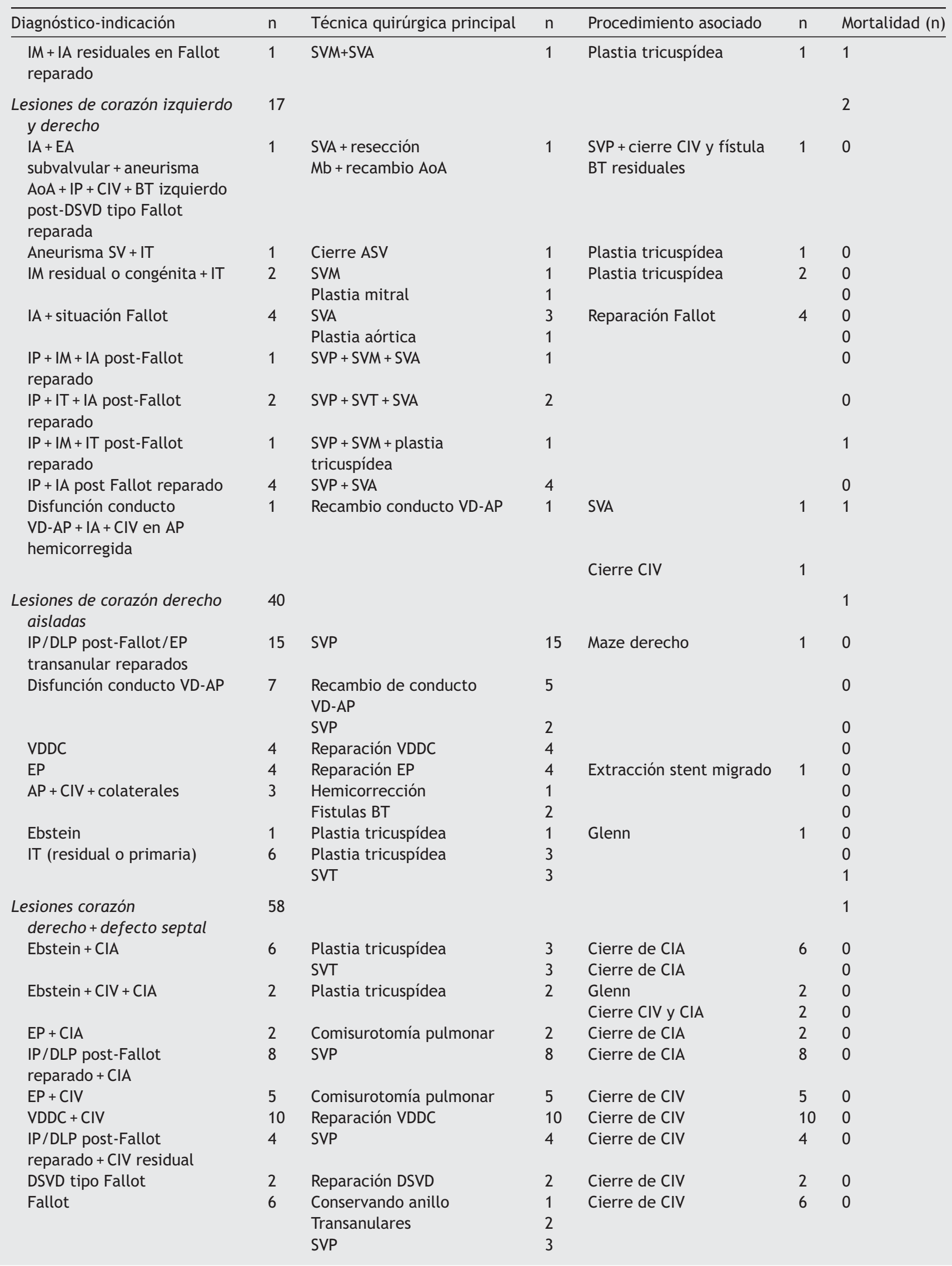


Tabla 2 (continuación)

\begin{tabular}{|c|c|c|c|c|c|c|}
\hline Diagnóstico-indicación & $\mathrm{n}$ & Técnica quirúrgica principal & $\mathrm{n}$ & Procedimiento asociado & $\mathrm{n}$ & Mortalidad (n) \\
\hline $\mathrm{IT}($ residual) + CIA & 10 & Plastia tricuspídea & 10 & Cierre de $\mathrm{CIA}$ & 10 & 0 \\
\hline IT (residual) + CIV & 3 & Plastia tricuspídea & 3 & Cierre de CIV & 3 & 0 \\
\hline $\begin{array}{l}\text { Lesiones corazón derecho } \\
\text { a } 2 \text { niveles }\end{array}$ & 15 & & & & & 1 \\
\hline $\begin{array}{l}\text { IP + hipoplasia VD post-EP } \\
\text { transanular }\end{array}$ & 1 & Conducto VD-AP & 1 & Glenn & 1 & 1 \\
\hline DSVD tipo Fallot + IT & 1 & Reparación DSVD & 1 & Plastia tricuspídea & 1 & 0 \\
\hline $\begin{array}{l}\text { IP + IT post-Fallot/EP } \\
\text { transanular }\end{array}$ & 10 & SVP & 10 & Plastia tricuspídea & 10 & 0 \\
\hline $\begin{array}{l}\text { Disfunción prótesis pulmonar } \\
\text { y tricuspídea } \\
\text { Post-Fallot + flúter }\end{array}$ & 1 & RSVP & 1 & RSVT + Maze derecho & 1 & 0 \\
\hline $\begin{array}{l}\text { Disfunción conducto } \\
\text { VD-AP + DLT en APSI + flúter }\end{array}$ & 1 & RSVP & 1 & SVT + Maze derecho & & \\
\hline $\begin{array}{l}\text { Disfunción conducto VD } \\
\text { AP + EP subvalvular }\end{array}$ & 1 & Recambio conducto & 1 & $\begin{array}{l}\text { Ampliación vía salida } \\
\text { derecha nativa }\end{array}$ & 1 & 0 \\
\hline
\end{tabular}

Al: aurícula izquierda; AMI: arteria mamaria interna; Ao: aorta; AoA: aorta ascendente; AoD: aorta descendente; AP: arteria pulmonar/atresia pulmonar; APSI: atresia pulmonar con septo intacto; ASV: aneurisma seno de Valsalva; AV: auriculoventricular; BT: Blalock-Taussig; CEC: circulación extracorpórea; CIA: comunicación interauricular; CIV: comunicación interventricular; CoA: coartación de aorta; DAP: ductus arterioso persistente; DLA: doble lesión aórtica; DLP: doble lesión pulmonar; DPSAV: defecto parcial septo auriculoventricular; DSAV: defecto septo auriculoventricular; DSVD: doble salida de ventrículo derecho; DVPAP: drenaje venoso pulmonar anómalo parcial; DVPAT: drenaje venoso pulmonar anómalo total; EA: estenosis aórtica; ECMO: oxigenación extracorpórea de membrana; EM: estenosis mitral; EP: estenosis pulmonar; FOP: foramen oval permeable; IA: insuficiencia aórtica; IM: insuficiencia mitral; IP: insuficiencia pulmonar; IT: insuficiencia tricuspídea; Mb: membrana subaórtica; MP: marcapasos; RSVP: recambio prótesis pulmonar; RSVT: recambio prótesis tricuspídea; SV: sustitución valvular; SVA: sustitución valvular aórtica; SVM: sustitución valvular mitral; SVP: sustitución valvular pulmonar; SVT: sustitución valvular tricuspídea; TGA: transposición de grandes arterias; VD: ventrículo derecho; VDDC: ventrículo derecho de doble cámara; VI: ventrículo izquierdo.

Tabla 3 Asociación de la mortalidad quirúrgica con las variables estudiadas. Prueba de la $\chi^{2}$

\begin{tabular}{|c|c|c|c|}
\hline$n$ & Categorías & Mortalidad (\%) & $\mathrm{p}$ \\
\hline \multicolumn{4}{|c|}{ Reintervenciones } \\
\hline \multirow[t]{3}{*}{463} & Ninguna & 2.4 & $<0.001$ \\
\hline & 102 & 4.7 & \\
\hline & 3 o más & 21.1 & \\
\hline \multicolumn{4}{|c|}{ RACHS- 1} \\
\hline \multirow[t]{4}{*}{460} & 1 & 0.0 & $<0.001$ \\
\hline & 2 & 3.8 & \\
\hline & 3 & 6.8 & \\
\hline & 4 & 50 & \\
\hline \multicolumn{4}{|c|}{ Aristóteles } \\
\hline \multirow[t]{3}{*}{459} & Hasta 6 & 0.6 & 0.003 \\
\hline & De 6 a 9 & 5 & \\
\hline & 9 o más & 14.3 & \\
\hline \multicolumn{4}{|c|}{ Actividad quirúrgica } \\
\hline \multirow[t]{2}{*}{355} & Congénitas & 4.2 & 0.041 \\
\hline & Adquiridas & 6.8 & \\
\hline \multicolumn{4}{|l|}{ Sexo } \\
\hline \multirow[t]{2}{*}{463} & Varón & 3.3 & 0.513 \\
\hline & Mujer & 4.4 & \\
\hline
\end{tabular}

pacientes con relación a los cirujanos de plantilla representa la realidad de la mayoría de los servicios de cirugía cardíaca del sur de Europa. Supone un $8 \%$ de la actividad del quirófano de adultos y un porcentaje variable de la actividad asistencial de los cirujanos (un 18\% en el caso del de máxima dedicación, con actividad habitual en tratamiento de cardiopatías congénitas en la edad infantil). Sin embargo, el número anual de casos intervenidos permanece estable entorno a 25, a pesar del incremento del número de pacientes en seguimiento en consulta de cardiología de cardiópatas congénitos adultos, que ya superan los 3,800. Como Srinathan et al. ${ }^{9}$ y Klcovansky et al. ${ }^{10}$, lo atribuimos al importante aumento de los procedimientos hemodinámicos intervencionistas.

Son múltiples las formas de clasificar este heterogéneo grupo de enfermos. Hemos optado por una variante de la dinámica propuesta a la hora de enviar datos al Registro Europeo de Cirugía Cardiotorácica Congénita.

Los resultados de la aplicación de la escala de riesgo quirúrgico RACHS-1 ${ }^{16}$ y la de complejidad Aristóteles básico ${ }^{17}$ deben interpretarse con prudencia. La primera fue elaborada con pacientes menores de 18 años, y la segunda con niños y adolescentes. En ausencia de escalas de riesgo y complejidad propias de congénitos adultos, la validez de estos datos está por determinar.

Las cirugías más habituales (defecto septal primario/residual a nivel auricular con o sin drenaje venoso pulmonar anómalo asociado, defecto septal primario/residual 
Tabla 4 Asociación de la actividad habitual de los cirujanos con las características de la intervención

\begin{tabular}{lccr}
\hline & Cirujano de cardiopatías congénitas & Cirujano de cardiopatías adquiridas & $P$ \\
\hline RACHS-1 (media) & 2.38 & 2.27 & $0.282^{\mathrm{a}}$ \\
Aristóteles (media) & 6.90 & 6.46 & $0.036^{\mathrm{a}}$ \\
Reintervención (\%) & 55.5 & 29.1 & $<0.001^{\mathrm{b}}$ \\
\hline
\end{tabular}

a Prueba de la $t$ de Student.

b Prueba de la $\chi^{2}$.

Tabla 5 Modelo multivariante para la asociación de la mortalidad quirúrgica con factores de riesgo quirúrgico*

\begin{tabular}{llll}
\hline & OR & (IC 95\%) & $P$ \\
\hline Escala Aristóteles & 1.64 & $(1.18-2.29)$ & 0.003 \\
Tres o más intervenciones previas & 5.02 & $(1.44-17.52)$ & 0.011 \\
\hline
\end{tabular}

IC: intervalo de confianza; odds ratio.

"Ajustado por sexo.

Tabla 6 Modelo multivariante para la asociación de la mortalidad quirúrgica con el ámbito quirúrgico habitual de los cirujanos (congénitas o no congénitas)*

\begin{tabular}{lllr}
\hline & OR & (IC 95\%) & $P$ \\
\hline Cirujano de cardiopatías adquiridas & 3.53 & $(1.14-10.98)$ & 0.029 \\
Tiempo de circulación extracorpórea (fracciones de 10 min) & 1.13 & $(1.07-1.19)$ & $<0.001$ \\
\hline
\end{tabular}

IC: intervalo de confianza; OR: odds ratio.

"Ajustado por sexo.

a nivel ventricular o el implante de bioprótesis pulmonar \pm plastia tricuspídea por insuficiencia pulmonar residual tras reparación de Fallot o estenosis pulmonar congénita) se realizan sin o con muy baja mortalidad ${ }^{18,19}$.

Las reintervenciones por recambio de conducto disfuncionante suponen un paso más en el riesgo y en la complejidad (RACHS-3 y Aristóteles 8). Son frecuentes las adherencias retroesternales y el riesgo de rotura de cámaras cardíacas. Electivamente se establece CEC con acceso vascular periférico femoral o axilar antes de la reesternotomía si las pruebas de imagen indican situación de alto riesgo.

En los últimos 10 años comienzan a acumularse reintervenciones complejas de raíz aórtica secundarias a fracasos de cirugía de Ross, combinación de válvula bicúspide + dilatación de raíz aórtica + coartación reparada o anulectasias en cardiopatías conotruncales. Se han tratado bien mediante el recambio de la raíz con reimplante coronario (cirugía de Bentall-Bono) asociando en algunos casos técnicas de ampliación de anillo aórtico posterior (Nicks, Manouguian) o anterior (Konno), bien reimplantando la válvula aórtica nativa en casos seleccionados (cirugía tipo David). La mortalidad de esta cirugía compleja, en ocasiones con algún procedimiento asociado en el lado derecho del corazón, como puede ser el implante de una prótesis pulmonar o el recambio de un conducto entre el ventrículo derecho y la arteria pulmonar, ha sido del $9 \%$.

Tabla 7 Morbilidad perioperatoria

\begin{tabular}{lrr}
\hline Tipo de complicación & $\mathrm{n}$ & $\%$ \\
\hline Reintervención mayor durante el ingreso por lesión residual & 6 & 1.3 \\
Arritmia paroxística o definitiva supraventricular $^{\text {Infección hospitalaria }}$ & 17 & 3.7 \\
Drenaje torácico o pericárdico posquirúrgico tardío & 7 & 2.4 \\
Reintervención por sangrado & 6 & 1.5 \\
Accidente cerebrovascular con secuelas & 5 & 1.3 \\
Necesidad de marcapasos definitivo por bloqueo cardíaco completo & 5 & 1.1 \\
Parálisis frénica/recurrencial & 5 & 1.1 \\
Infarto cardíaco agudo perioperatorio & 4 & 1.1 \\
Insuficiencia renal transitoria con necesidad de hemodiafiltración & 2 & 0.9 \\
Asistencia mecánica circulatoria & 1 & 0.4 \\
\hline
\end{tabular}

\footnotetext{
a Infección hospitalaria: neumonía, sepsis, endocarditis resuelta mediante tratamiento antibiótico exclusivamente, infección de herida
} quirúrgica superficial, mediastinitis aguda, infección urinaria. 
Los peores resultados se produjeron en complicaciones posquirúrgicas como seudoaneurismas micóticos, de obligada reintervención; en la cirugía de la $D$ o $L$ transposición de grandes arterias en el adulto o en la del ventrículo único, casi todos multiintervenidos y cianóticos ${ }^{20}$. Consideramos que en estos 2 últimos grupos de pacientes, por su dificultad técnica, elevada mortalidad y escasa mejoría funcional, debe valorarse con sumo cuidado la relación riesgo-beneficio de la cirugía ${ }^{21,22}$.

La mortalidad se asoció con el tiempo de CEC, pero no con el de ClAo, probablemente porque algunas cirugías sobre el corazón derecho sin defecto septal asociado (hasta 33 intervenciones) se realizaron sin isquemia miocárdica, con el corazón latiendo o fibrilando, que consideramos la mejor protección miocárdica para el ventrículo derecho disfuncionante. De forma similar a lo descrito en otras series quirúrgicas congénitas, la mortalidad se asoció también con el riesgo quirúrgico y la complejidad de las intervenciones ${ }^{5,23}$.

Recientemente han aparecido publicaciones tratando de demostrar cuál es la combinación ideal (cirujanos de adquiridas o de congénitas operando en ámbito infantil o en un hospital general) que ofrece mejores resultados a los congénitos adultos, con recomendaciones dispares ${ }^{11-15}$. Nosotros solo hemos operado a los adultos en el hospital general, y en la serie completa de la UCCA sí resulta significativo como factor protector el hecho de ser intervenidos por cirujanos con actividad habitual en el ámbito de la congénita (a diferencia de lo que describíamos en el subgrupo de las reintervenciones en el año 2009, entonces al borde de la significación) $)^{23}$. Pensamos que, lejos de excluir, probablemente los resultados todavía tienen margen de mejora individualizando más la toma de decisiones en los pacientes más complejos, aprovechando la experiencia quirúrgica de cada equipo en técnicas muy específicas, de forma complementaria.

El hecho de que la mayor complejidad quirúrgica y el mayor porcentaje de reintervenciones se den entre los cirujanos con actividad habitual en cardiopatías congénitas, y aun así la mortalidad sea menor en las operaciones llevadas a cabo por estos cirujanos, refuerza la solidez de los resultados obtenidos.

En cuanto a la progresiva mejoría en la «calidad» de la serie desde 2001, atribuimos el resultado a cambios organizativos realizados entonces en el seno de la UCCA: sesiones médico-quirúrgicas semanales específicas para los congénitos adultos con la participación del hemodinamista y el arritmólogo con dedicación preferente a la problemática del adulto con cardiopatía congénita, coordinación quirúrgica por parte de un cirujano de congénitas, añadida a la clínica cardiológica ya existente en el organigrama de la unidad desde su origen, protagonismo definitivo de los cirujanos de congénita a la hora de realizar las intervenciones quirúrgicas, además de a la experiencia creciente de todo el personal que la componen.

\section{Conclusiones}

La cirugía del congénito adulto se ha realizado con buenos resultados en el intervalo estudiado, equiparables a lo publicado en series internacionales en cuanto a mortalidad hospitalaria y morbilidad perioperatoria. El número de intervenciones por año permanece estable a pesar del incremento en el número de pacientes en seguimiento cardiológico.

Factores asociados a mortalidad hospitalaria en análisis multivariante fueron el tiempo de CEC, las múltiples reintervenciones, la complejidad creciente y la intervención del paciente por parte de un cirujano con actividad habitual en cardiopatías adquiridas.

La reestructuración del funcionamiento de la Unidad desde 2001 y la experiencia acumulada influyen significativamente en los resultados, de modo que a pesar de aumentar la complejidad y el riesgo quirúrgico de los pacientes más modernos de la serie, la mortalidad ha disminuido.

\section{Financiación}

No se recibió patrocinio de ningún tipo para llevar a cabo este artículo.

\section{Conflicto de intereses}

Los autores declaran no tener ningún conflicto de intereses a la hora de elaborar este original.

\section{Bibliografía}

1. Webb GD, Williams RG. 32nd Bethesda Conference. Care of the adult with congenital heart disease: Introduction. J Am Coll Cardiol. 2001;37:1166-9.

2. Warnes CA, Liberthson R, Danielson GK, et al. 32nd Bethesda Conference. Task force 1: The changing profile of congenital heart disease in adult life. J Am Coll Cardiol. 2001;37:1170-5.

3. Oliver Ruiz JM. Cardiopatías congénitas del adulto: residuos, secuelas y complicaciones de las cardiopatías congénitas operadas en la infancia. Rev Esp Cardiol. 2003;56:73-88.

4. Bhat AH, Sahn BD. Congenital heart disease never goes away, even when it has been «treated»: The adult with congenital heart disease. Curr Opin Pediatr. 2004;16:500-7.

5. Berdat PA, Immer F, Pfammatter JP, et al. Reoperations in adults with congenital heart disease: Analysis of early outcome. Int $J$ Cardiol. 2004;93:239-45.

6. Monro JL, Alexiou C, Salmon AP, Qeeton BR. Reoperations and survival after primary repair of congenital heart defects in children. J Thorac Cardiovasc Surg. 2003;126:511-20.

7. Vida VL, Berggren H, Brawn W, et al. Risk of surgery for congenital heart disease in the adult: A multicentered European study. Ann Thorac Surg. 2007;83:161-8.

8. Rossano JW, OB́rian Smith E, Fraser Jr CD, et al. Adults undergoing cardiac surgery at a children' hospital: An analysis of perioperative morbidity. Ann Thorac Surg. 2007;83:606-12.

9. Srinathan SK, Bonser RS, Sethia B, et al. Changing practice of cardiac surgery in adult patients with congenital heart disease. Heart. 2004;91:207-12.

10. Klcovansky J, Sondergaad L, Helvind M, et al. Cardiac surgery in grown-up congenital heart patients. Will the surgical workload increase? Interact Cardiovasc Thorac Surg. 2008;7:84-9.

11. Karamlou T, Diggs BS, Person T, et al. Operation by pediatric heart surgeons decreases in-hospital death. National practice patterns for management of adult congenital heart disease. Circulation. 2008;118:2345-52.

12. Mahle WT, Kirshbom PM, Kanter KR, et al. Cardiac surgery in adults performed at children' hospitals: Trends and outcomes. J Thorac Cardiovasc Surg. 2008;136:307-11. 
13. Kogon BE, Plattner C, Leong $T$, et al. Adult congenital heart surgery: Adult or pediatric facility? Adult or pediatric surgeon? Ann Thorac Surg. 2009;87:833-40.

14. Karamlou T, Diggs BS, Ungerleider RM, et al. Adults or big kids: What is the ideal clinical environment for management of grown-up patients with congenital heart disease? Ann Thorac Surg. 2010;90:573-9.

15. Dearani JA. Surgery for adults with congenital heart disease should be performed by congenital heart surgeons. J Thorac Cardiovasc Surg. 2009;138:5-7.

16. Jenkins KJ, Gauvreau K, Newburguer JW, et al. Consensusbased method for risk adjustment for surgery for congenital heart disease. J Thorac Cardiovasc Surg. 2002;123: $110-8$.

17. Lacour-Gayet F, Clarke D, Jacobs J, et al. The Aristotle score for congenital heart surgery. Semin Thorac Cardiovasc Surg Pediatric Card Surg Ann. 2004;7:185-91.
18. Polo L, Aroca A, González A, et al. Cirugía del drenaje venoso pulmonar anómalo parcial en paciente adulto. Cir Cardiov. 2010;17:120.

19. Polo L, Aroca A, Cabestrero D, et al. Adult life reoperations in Fallot patients with corrective surgery during childhood. Cardiol Young. 2011;21 Suppl1:107S.

20. Aroca A, Polo L, González A, et al. Cirugía cavopulmonar en la situación de ventrículo único durante la edad adulta. Resultados quirúrgicos y seguimiento. Cir Cardiov. 2010;17:92.

21. Gatzoulis MA, Munk MD, Williams WG, et al. Definitive palliation with cavopulmonary or aortopulmonary shunts for adults with single ventricle physiology. Heart. 2000;3:51-7.

22. Veldtman GR, Nishimoto A, Siu S, et al. The Fontan procedure in adults. Heart. 2001;86:330-5.

23. Aroca A, Segovia J, Polo L, et al. Cardiopatías congénitas del adulto. Reintervenciones quirúrgicas y trasplante de órganos torácicos. Rev Esp Cardiol Supl. 2009;9:98E-107E. 